\title{
Home based care improved glycaemic control and was cost effective in children with type 1 diabetes
}

\author{
Dougherty G, Schiffrin A, White D, et al.Home-based management can achieve intensification cost-effectively in type 1 diabetes. \\ Pediatrics 1999 Jan;103:122-8.
}

\section{Question}

Is a home based care programme more effective for glycaemic control and more cost effective than conventional hospital based care in children with newly diagnosed type 1 diabetes mellitus?

\section{Design}

Randomised, unblinded, controlled trial with 3 years follow up.

\section{Setting}

An emergency department of a secondary and tertiary care paediatric teaching hospital in Montreal, Quebec, Canada.

\section{Patients}

63 consecutive children (mean age 10 y, $56 \%$ girls) who were newly diagnosed with type 1 diabetes, $>2$ years of age, living at home and within 1 hour of the hospital, and who had no sibling with type 1 diabetes. Follow up was 100\%.

\section{Intervention}

Within 72 hours of diagnosis, children were allocated to either home based care $(n=32)$ or hospital based care $(n=31)$. Children allocated to home based care had insulin treatment and teaching at home by a diabetes treatment nurse; were admitted to hospital for metabolic stabilisation if necessary; had flexibly scheduled, initial nurse visits once or twice daily; and a clinic teaching session. Children allocated to hospital based care remained in hospital for metabolic stabilisation and initial insulin treatment, and received teaching in the hospital. Children in both groups received similar information, had nurse home visits and telephone advice when needed, had insulin treatment, and were encouraged to do home glucose monitoring.

\section{Main outcome measures}

Haemoglobin $\left(\mathrm{HbA}_{1 \mathrm{c}}\right)$; diabetes related adverse events; diabetes knowledge (Diabetes Knowledge Scale), regimen adherence (Diabetes Regimen Adherence Questionnaire-R), family impact
(Impact on Family Scale), stress (Perceived Stress Scale), treatment satisfaction (Satisfaction Scale), child behaviour (Achenbach Child Behavior Checklist); and social costs (1991 Canadian dollars).

\section{Main results}

Children who received home based care had lower mean $\mathrm{HbA}_{1 c}$ values than children who received hospital based care at 2 years $(6.1 \%$ v $6.8 \%, \mathrm{p}<0.05)$ and 3 years $(6.4 \%$ v $7.1 \%, \mathrm{p}<0.02)$. No differences existed between groups for diabetes related adverse events at 2 years; parent measures (diabetes knowledge, regimen adherence, family impact, stress, satisfaction with treatment, and child behaviour); or adolescent measures (diabetes knowledge, regimen adherence, stress, and satisfaction with treatment) at 1 and 2 years. During the first month, parents of children who received home based care spent less time (mean difference $52 \mathrm{~h}$, $\mathrm{p}<0.001$ ) and less money on treatment related expenses (mean difference $\mathrm{CN} \$ 100.53)$ than parents of children who received hospital based care. Social costs did not differ between groups in the first 2 years after diagnosis and were $\$ 48$ higher for each child with home based care when parents' time was valued at $\$ 11.88 /$ hour.

\section{Conclusion}

In children with newly diagnosed type 1 diabetes, home based care improved glycaemic control and had similar knowledge, adherence, and psychosocial effects compared with hospital based care, and was cost effective (social perspective).

Source of funding: National Health Research and Development Program of Canada.

For correspondence: Dr G Dougherty, The Montreal Children's Hospital, 2300 Tupper Street, Room A-216, Montreal, Quebec H3H 1P3, Canada. Fax +1 5149344424 .

\section{Commentary}

The Diabetes Control and Complications Trial (DCCT) recommended intensive management of type 1 diabetes mellitus in most children and adolescents, ${ }^{1}$ but little is known about strategies for implementation in youth. Home based care has been shown to be effective without increasing overall costs. ${ }^{2}$

This study by Dougherty et al used a randomised controlled trial design, which provides the least biased evaluation of an intervention. However, questions remain about the generalisability of the findings to other groups. The study participants represent a more affluent group than might be expected in general practice.

The findings of the study are impressive. The home based group's improved $\mathrm{HbA}_{\mathrm{lc}}$ after 2 years corresponds to a $10-25 \%$ reduction in the incidence of microvascular complications found in the
DCCT, ${ }^{1}$ which makes the difference clinically significant. This difference occurred despite the fact that the interventions were quite similar. It is possible that the home based group achieved this level of improved control because decisions were based on information gathered from a child's everyday life experience rather than information gathered from a child's hospital stay. Another strength of the study is the use of outcomes other than metabolic control, such as knowledge, adherence, psychosocial effects, and costs.

Paediatric nurses and advanced practice nurses working with children with type 1 diabetes will find the findings relevant to their practice. The findings reinforce the idea that clinical decisions based on a child's usual food and activity patterns are likely to be better than those based on the artificial hospital environ- ment. Inpatient management should probably be reserved for children who require more intensive medical intervention, such as those in the acute phase of diabetic ketoacidosis.

Margaret Grey, CPNP, DrPH
Independence Foundation
Professor of Nursing
Associate Dean for Research Affairs
Yale School of Nursing
New Haven, Connecticut, USA

1 Diabetes Control and Complications Trial Research Group. Effect of intensive diabetes treatment on the development and progression of long-term complications in adolescents with insulin-dependent diabetes mellitus. Diabetes control and complications trial. $J$ Pediatrics 1994;125:177-88.

2 Brooten D, Naylor MD. Nurses' effect on changing patient outcomes. Image J Nurs Sch 1995;27:95-9. 